\title{
On Transaction Boundaries in Active Databases: A Performance Perspective
}

\author{
Michael J. Carey, Member, IEEE, Rajiv Jauhari, Member, IEEE, and Miron Livny
}

\begin{abstract}
The workload of an active DBMS consists of two types of activities: externally generated tasks submitted by users, and rule management tasks caused by the triggering of rules stored in the knowledge component of the system. Most design proposals for active DBMS's assume that an external task should be combined with all the resulting rule management tasks into a single transaction. There is no compelling reason for this assumption, however; the semantics of the rules can be used to divide the workload into transactions in a number of different ways. In this paper, we describe a performance model designed for studying active DBMS performance issues. We present the results of simulation experiments that study system performance as a function of transaction boundary semantics for varying levels of data contention, rule complexity, and data sharing between externally submitted tasks and rule management tasks. Our results demonstrate that the way in which transaction boundaries are imposed can have a major impact on the performance of an active DBMS. We therefore conclude that this aspect of rule semantics must be carefully considered at the time that rules are specified.
\end{abstract}

Index Terms-Active databases, database management, performance evaluation, rule systems, transaction management.

\section{INTRODUCTION}

$\mathbf{I}_{d}^{1}$ $\mathrm{T}$ is generally recognized that in order to meet the growing demands placed on information processing systems, rules and facts need to be integrated into a "knowledge base management" framework. A computer-controlled factory, for example, can be expected to take advantage of knowledge base management in a number of areas. The inventory of the factory will be controlled automatically by rules that trigger "ordering" actions when the facts of the system indicate a low "quantity-on-hand" of some part. The beginning of each step of the manufacturing process will be triggered by a rule that recognizes the end of the preceding step. Facts regarding the structural properties of the products will be measured and recorded as the products pass through the manufacturing line, and defective items will be identified with the help of rules that trigger actions to discard such items. The "technical secrets" of

Manuscript received November 23, 1988; revised February 15, 1990. This work was performed under subcontract to Xerox Advanced Information Technology, which was supported by the Defense Advanced Research Projects Agency and by the Rome Air Development Center under Contract F30602-87C-0029. Additional support was provided by the National Science Foundation under Grant IRI-8657323 and by the Digital Equipment Corporation through its Incentives for Excellence program. The views and conclusions contained in this paper are those of the authors and do not necessarily represent the official policies of the Defense Advanced Research Projects Agency, the Rome Air Development Center, or the U.S. Government.

The authors are with the Computer Sciences Department, University of Wisconsin, Madison, WI 53706.

IEEE Log Number 9101531 the manufacturing process may be kept in a secure file to which access is restricted via rules, with the additional safeguard that the names of all those who access the file are automatically logged.

Rules and facts can be integrated in a number of ways. One way to structure a knowledge base management system is to couple a rule manager and a database management system (DBMS) loosely via a polling mechanism. The rule manager, which appears as just another user to the DBMS, periodically runs queries to monitor the facts stored in the DBMS. Depending on its rules, update transactions may also submitted by the rule manager. It has been argued in [32] that such a loosely-coupled system is likely to perform poorly (or even incorrectly) unless the application has specific characteristics - for example, that the rules need to access only a small part of the data, and that the data remain unchanged while they are being processed by the rules. Most large-scale applications of knowledge-based data management (such as factory control, air traffic control, and stock trading) seem not to have these characteristics, and thus require a tighter coupling between the rules and the facts. Such applications have motivated the development of a number of active DBMS's (e.g., [19], [25], [28], [33], [23]), in which rules and facts are tightly-coupled. In these systems, data and rules are both managed by the DBMS using an integrated language and data model.

The workload of an active DBMS is a mix of External Tasks (ET's) and Rule Management Tasks (RMT's). ET's are queries and updates that arrive at the active DBMS from a user or an application program, whereas tasks that result from the presence of rules (both condition-checking tasks and responses to satisfied conditions) constitute the RMT's of the active DBMS. ET's may trigger RMT's, which may in turn trigger other RMT's. We call the set of tasks executed as a consequence of the arrival of a single ET (including the ET itself) a job. The time taken by the DBMS to complete a job is a measure of the speed with which the system reacts to external stimuli; thus, job response time is a new performance metric of interest when evaluating the behavior of an active DBMS.

Clearly, the tasks that make up a job must be combined in some fashion to form transactions. In most of the proposals for active DBMS's that have appeared in the literature (e.g., [25], [33]), the user has little flexibility in determining transaction boundaries. For example, a rule in POSTGRES [33] might state that the values of two data items $d_{1}$ and $d_{2}$ are required to be equal. In this case, an update to $d_{1}$ will cause $d_{2}$ to be automatically updated as well; the automatic $d_{2}$ update will take place either when $d_{1}$ is updated ("early evaluation") 
or else when $d_{2}$ is subsequently accessed ("late evaluation"). Thus, in POSTGRES, the RMT will be coupled either to the updating ET or to the accessing ET, depending on the evaluation scheme used. As demonstrated by the example in the Appendix, the semantics of rules do not always require such strict coupling of the ET and the RMT's of a job.

In the general case, the relationship between jobs and transactions is that, while a job may consist of one or more transactions, a transaction can be part of at most one job. The simplest mapping of jobs onto transactions is one-toone, with all the tasks of a job being coupled into a single transaction, as in POSTGRES. An alternative mapping scheme could, for example, allow each task of a job be an independent transaction. When the semantics of a rule allow multiple possible mappings between jobs and transactions, the relative performance provided by the alternative mapping schemes becomes an issue of interest. If there are significant performance differences between the mapping schemes, it may be useful to provide the flexibility of choosing among a set of task-transaction coupling modes for each rule in the active DBMS, as in [10]. A quantitative analysis of the performance of alternative coupling modes would then be of interest to rule designers.

The decoupling of a job into smaller transactions may affect system performance in two ways. If there are significant fixed costs associated with transaction startup and transaction commit, increasing the number of transactions in this way could lead to worse performance. On the other hand, from a concurrency control perspective, decoupling a job may result in shorter average job response times, especially when data contention becomes significant. For example, if locking is used for concurrency control, the average duration for which locks are held will decrease as transactions are made smaller by decoupling jobs. This will reduce lock contention, even though the total amount of data accessed per job will remain the same.

From a performance viewpoint, several issues need to be explored with regard to the coupling of tasks to form transactions. First, even though job decoupling may result in improved job response times if the fixed costs of transaction startup and commit are small, how significant is this improvement likely to be? Second, and more interestingly, the data access characteristics of ET's and RMT's may differ (e.g., RMT's that check conditions are likely to be read-only queries, while ET's and RMT's triggered in response to satisfied conditions may include updates). Can these differences in data access characteristics significantly influence the way in which task coupling affects job response time? Finally, how do these differences in data access characteristics affect the relative performance of different types of task-transaction coupling methods as task complexity increases? These are some of the issues examined in this paper.

In order to help us investigate these issues, we have developed a performance model of an active DBMS where the triggering of rules is modeled via probabilities. This probabilistic model provides a platform that can be used to investigate performance issues in active database systems without having to explicitly model the rules and the data. The execution and knowledge models developed as part the High-
Performance Active (HiPAC) Database project [10] were used to guide the design of our model. The goal of the HiPAC project was to design an active, time-constrained, objectoriented database system. The execution model of HiPAC provides a number of different coupling modes that allow the user to specify the way in which ET's and RMT's should be combined into transactions; the coupling mode is considered part of a rule and is specified at the time the rule is created.

The remainder of the paper is organized in the following manner. Section II surveys related work in the area of active database systems, including a brief overview of the HiPAC project. We discuss our model of transaction processing in an active DBMS in Section III. Our probabilistic model of an active DBMS is described in Section IV. In Section V, our experiments and their results are discussed. Section VI contains our conclusions and a brief discussion of our continuing work in related areas. Finally, a detailed example showing how ET's and RMT's can be combined to form transactions based on rule semantics is presented in the Appendix.

\section{RELATED WORK}

Little work has been done in the area of evaluating the performance of knowledge-based data management systems. However, the need for integrating rules and facts in a DBMS context has been recognized for a long time. It has been argued that the lack of a rule facility can place a significant burden on the DBMS application programmer. For example, in order to support general integrity constraints in the absence of such a facility, every transaction that updates the database for a given application must be augmented with code to check the constraints and to take an appropriate action if a constraint is violated. Thus, the condition checking activity and the action become integral parts of users' transactions. In recent years, various approaches have been suggested for adding active capabilities to database systems in order to integrate rules and facts and thus simplify the application programming task. We briefly review some of the major approaches in the rest of this section.

The addition of active capabilities to database systems was first considered in order to support specific DBMS functions such as view maintenance and integrity constraint enforcement. It was proposed in [12] and [13] that "triggers" be added to System $\mathrm{R}$ in order to enforce integrity constraints or "assertions." Triggering mechanisms of different types have also been suggested to support the maintenance of materialized views, snapshots, and derived attribute values [6], [27], [24], [4], [20], [15], [3], [17]. More recently, it has been proposed that generalized active data management capabilities be added to database systems in order to provide a unified mechanism to support a variety of applications, such as those requiring rulebased inferencing [30], [19], [25], [28], [35]. The POSTGRES project [31], [33] proposes a general mechanism to support alerters, triggers, and rules (among other features) in the context of an extended relational data model.

The HiPAC project combines active, object-oriented databases with time constraints [10]. In the HiPAC knowledge model [9], rules are treated as an object class, like all other 
forms of data. Each rule in HiPAC is structured according to the event-condition-action (ECA) paradigm. The event associated with a rule is the cause of the rule being activated, such as an update to the "quantity-on-hand" object set in the factory control example discussed earlier. The condition is a set of queries which must be evaluated in order to check whether a prespecified set of predicates has been satisfied. If cars were assembled in the factory in our example, a condition could be a query representing the question "is the number of radios in stock low enough that we need to order more?" Finally, the action represents the operations to be executed if the condition is met, for example, an order for a thousand radios could be sent automatically to a radio supplier. As mentioned earlier, the way that the event, condition, and action of a rule are to be combined into transactions is specified as part of the definition of a rule in HiPAC. HiPAC's transaction execution model, described in [16], is based on a nested transaction model. Different methods are proposed for mapping events, conditions, and actions onto transactions and subtransactions. A subset of these methods is included in the execution model used in this paper, as described shortly.

\section{Transaction Model for Active Databases}

As discussed earlier, an active database system contains a set of rules in addition to the data or facts found in all database systems. These rules can create Rule Management Tasks (RMT's) in response to External Tasks (ET's) that represent external stimuli. The semantics of the rules determine the way in which RMT's can be combined with ET's to form transactions. In this section, we describe our model of transaction execution in an active DBMS.

\section{A. External Tasks, Checkers, and Actions}

As stated in Section I, a job is the sum total of all the database activity generated by an incoming External Task. Of course, the minimum amount of work represented by a job consists of the initial External Task itself. In terms of the ECA paradigm, External Tasks can cause Events. An Event in the ECA model, however, is defined as part of a rule; there may be user queries and updates whose activity does not correspond to the Event of any rule defined in the system. When an External Task causes an Event, the job will also include a Checker task. Checkers are system-generated queries that check whether the conditions of a rule are satisfied. The Checker will generate an Action task to perform the operation dictated by the rule if the conditions are met. Since an Action may query or update the database, it may cause Events that in turn lead to further Checkers. In this way, a single External Task could cause an arbitrarily long chain of tasks, all of which would be part of the same job.

\section{B. Coupling Tasks to Form Transactions}

As discussed in Section I, the tasks of a job need to be combined to form transactions in some way, and both rule semantics and performance effects may need to be considered when making the mapping from tasks to transactions. The imposition of a transaction structure on a job suggests the need for a nested transaction model, such as the model proposed in [16]. In this model, there are two types of choices to be made when scheduling an RMT. The first choice involves transaction boundaries: whether the RMT is required to be part of the same transaction as the task which generated it, or instead can be executed in a separate transaction. The second choice determines the time at which the RMT should begin execution: for example, should a Checker begin execution as soon as the triggering event takes place, or should it begin execution only when the triggering task completes? In the work presented here, we study the performance implications of the first of these two choices: the transaction boundary decision. In order to do so, we selected a subset of the transaction execution model described in [16] as our execution model. In our execution model, any internally generated activity caused by task $T$ of a job $J$ is started only after the causing task is "completed." A task $T$ is said to have completed at the time when, had $T$ been a transaction by itself, it would have been in a position to commit. Thus, our execution model fixes the timing of an RMT relative to its triggering task, enabling us to isolate the performance effects of the transaction boundary decision from the effects of the timing decision.

Our execution model supports four different ways in which the tasks of a job can be coupled to form transactions:

1) Strict Coupling ( $S$-Coupling): The ET of the job and the RMT's of each rule activated are coupled together in a single transaction. In effect, all the tasks of a job are part of a single transaction.

2) External Task-Checker Coupling (EC-Coupling): The ET of the job is coupled into a single transaction with the Checkers that it generates, but each Action generated by a Checker begins a new transaction. Recursively generated Checkers are coupled with their corresponding causing Actions. ${ }^{1}$

3) Checker-Action Coupling (CA-Coupling): The ET of the job is a stand-alone transaction, but every Checker is coupled with the corresponding Action into a single transaction. Each Checker begins a new transaction.

4) No Coupling ( $N$-Coupling): Each task of the job exists as a stand-alone transaction.

The following example illustrates the differences between these four coupling modes. Job $J$ begins when External Task $E$ arrives at the system. $E$ triggers a rule $R_{1}$, which results in Checker $C_{1}$ being executed. The condition checked by $C_{1}$ is found to be satisfied, and an Action $A_{1}$ results. In turn, $A_{1}$ activates rule $R_{2}$ and causes another Checker $C_{2}$ to be run. $C_{2}$ leads to yet another Action $A_{2}$. Finally, $A_{2}$ does not trigger any further rules, so job $J$ consists of a total of five tasks: one ET and four RMT's. Table I shows how the four coupling modes would combine the tasks of job $J$ into transactions; to keep the example simple, we have assumed that $R_{1}$ and $R_{2}$ have the same transaction coupling semantics. If S-coupling were employed, job $J$ would consist of exactly one transaction. If either EC-coupling or CA-coupling were used, there would be three transactions, although the transaction boundaries would

\footnotetext{
${ }^{1}$ The ODE project [3] at Bell Labs recently adopted EC-coupling for use in their system's trigger facility.
} 
TABLE I

TRANSACTION BOUNDARIES FOR JOB $J$

\begin{tabular}{llll}
\hline Coupling Mode & $\begin{array}{c}\text { Transaction Boundaries } \\
\text { ("[ } \ldots] \text { " is a transaction) }\end{array}$ & $\begin{array}{c}\text { Number of } \\
\text { Transactions }\end{array}$ \\
\hline S-coupling & {$\left[\begin{array}{lllll}E & C_{1} & A_{1} & C_{2} & A_{2}\end{array}\right]$} & 1 \\
EC-coupling & {$\left[\begin{array}{llll}E & C_{1}\end{array}\right]\left[\begin{array}{lll}A_{1} & C_{2} & {\left[A_{2}\right.}\end{array}\right]$} & 3 \\
CA-coupling & {$[E]\left[\begin{array}{lll}C_{1} & \left.A_{1}\right]\left[C_{2}\right. & A_{2}\end{array}\right]$} & 3 \\
N-coupling & {$[E]\left[C_{1}\right]\left[A_{1}\right]\left[C_{2}\right]\left[A_{2}\right]$} & 5 \\
\hline
\end{tabular}

differ as shown in Table I. If $\mathrm{N}$-coupling were used, each of job $J$ 's tasks would be its own transaction. In the Appendix, we present an example that illustrates the use of these coupling modes and discusses some of the associated tradeoffs.

The coupling of tasks into transactions requires a modification of conventional transaction restart semantics. In our model of an active DBMS, Checkers and Actions occur only in response to changes in the state of the data. If a transaction begins with an External Task, then the External Task must clearly be reexecuted when the transaction is restarted. If a transaction begins with a rule Management Task, this means that the DBMS has recognized a change of state that requires the execution of a Checker or an Action. Thus, the system must guarantee that the initial RMT is repeated if the transaction is restarted. In our execution model, then, whenever a transaction aborts, it is restarted at the first task (External Task, Checker, or Action) that formed part of the aborted transaction. The subsequent tasks may or may not be repeated, as the state of the database may have been changed by updates of other transactions that ran since the aborted transaction began.

\section{Modeling AN ACTIVE DBMS}

Our performance model of an active DBMS is an extension of the model of a database site used in the distributed database study described in [7]. It has been implemented using the DeNet simulation language [22], so it can easily be extended later to include multiple sites in order to study a distributed active DBMS.

In our model, an active DBMS has five components: a Source, which generates the External Tasks; a Transaction Manager, which models the execution behavior of transactions; a Concurrency Control Manager, which implements the details of a particular concurrency control algorithm; a Resource Manager, which models the CPU, I/O, and main memory resources of the system; and finally, a Rule Manager, which models the active component of the database. Fig. 1 presents a detailed view of these components and their key interactions. The model described in [7] already contained the first four of these components. However, these components have been modified in our model to capture the active nature of the workload. Also, certain additional features (such as a buffer manager) have been included in our model. Of course, the major extension to the original model is the inclusion of the Rule Manager component. In this section, we first describe the passive database model. The Rule Manager module is then described in detail. Finally, the remaining modules and their parameters are briefly described.

\section{A. Modeling the Passive Database}

The passive portion of the database is modeled simply as a collection of files. In turn, each file is modeled as a collection of pages. The parameters of the database model include NumFiles, which is the number of files in the database, and FileSize ${ }_{i}$, the number of pages in each file $(1 \leq i \leq$ NumFiles). Each file can be viewed as representing a set of database objects; we model files at the page level because we assume page-oriented concurrency control for simplicity.

\section{B. The Rule Manager Module}

The Rule Manager models the active portion of the database and the active functions of the DBMS. In terms of the execution model, the Rule Manager must capture the rules in the active portion of the database, and it has to interact with the Transaction Manager in order to coordinate the execution of External Tasks, Checkers, and Actions. Table II lists the input parameters for the rule manager. A detailed description of the Rule Manager module follows.

1) Modeling the Active Database: We use probabilities to model the occurrence of Events, Conditions, and Actions. This probabilistic approach to the modeling of the active DBMS was chosen for two reasons. First, in order to have as general a model as possible, we tried to minimize the amount of specific information maintained by the simulation model, while still modeling the system at a level appropriate for a first performance study of an active DBMS. For example, in order to study the impact of an increased RMT workload on the relative performance of the different coupling modes discussed in Section III-B, we simply change the probability of occurrence of Checkers and Actions; had we modeled the rules explicitly, we would have had to change the "condition" parts of some or all of the rules (or changed the details of the ET workload). Second, explicit modeling of the rules would have required explicit modeling of the data (including such details as the values of data objects) and the workload; this would have made our simulations computationally expensive without adding significantly to our understanding of the task-transaction mapping issue.

To further simplify the active workload model, we assume here that a task can trigger at most one rule. The probability that an External Task leads to a Checker is ExtCheckProb. Similarly, the probability that the condition of a rule is satisfied (i.e., that an Action needs to be scheduled after a Checker) is ActionProb. Finally, the probability of an Action leading to another Checker is ActCheckProb. The coupling mode of a task (i.e., the way that it is coupled with the task that preceded it) is specified via the CouplingTypeProb parameter array. CouplingTypeProb has four entries, each representing the probability of using one of the four coupling modes described in Section III-B. Note that our assumption that each task can trigger at most one rule leads to a single sequential thread of tasks for each job, and that the tasks occur in the following order:

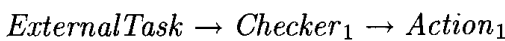

$$
\begin{aligned}
& \rightarrow \text { Checker }_{2} \rightarrow \text { Action }_{2} \rightarrow \cdots \text {. }
\end{aligned}
$$


3) Modeling Open-Ended Transaction Execution: The Rule Manager coordinates the execution of active transactions with the Transaction Manager. Consider the case of a nearlycompleted External Task as an example. When an External Task $E$ has completed its reads and updates successfully, and has reached the point where it can commit, the Rule Manager is invoked to check whether $E$ 's transaction can be committed. First, the Rule Manager determines whether any rule has been triggered by $E$. If a rule has been triggered, its Checker must be scheduled; the Rule Manager then decides the coupling mode to use. Depending on the input parameters, the Rule Manager responds in one of the following ways:

1) If the Rule Manager determines that the Checker is to be coupled with $E$ 's transaction, it initializes a new task which is appended to $E$ 's transaction.

2) If the Rule Manager determines that the Checker must be scheduled as part of a different transaction, it initializes a new task and directs the Transaction Manager to execute the new task as an independent transaction. In this case, $E$ 's transaction is allowed to commit, while $E$ 's job remains active.

If $E$ did not trigger any rule, the Rule Manager informs the Transaction Manager that $E$ 's job has completed execution, and that $E$ 's transaction may be committed. The Rule Manager and the Transaction Manager interact in a similar fashion when either a Checker or an Action reaches the point where it can commit.

4) Modeling Restarts: The Transaction Manager is responsible for informing the Rule Manager of each transaction abort. When it receives the information about an abort, the Rule Manager directs the Transaction Manager to restart the first task which was part of the aborted transaction. In our performance model, since decisions are made probabilistically, this restart mechanism can unfortunately lead to a bias against long jobs. Consider a system where there is high contention for data and all jobs are strictly coupled. Suppose a job $J$ begins with a External Task task $E$, goes through a Checker task $C$, and is in Action task $A$ when it is aborted. It will then be restarted beginning at task $E$. Let us assume that it completes task $E$ again and is then aborted in task $C$. It will then once again begin with task $E$. If the decisions to schedule $C$ and $A$ are made entirely on the basis of probabilities, it is more likely that job $J$ will ultimately complete execution if it consists of a single task rather than two or three S-coupled tasks. Thus, there will be a tendency to favor shorter jobs over longer ones, i.e., restarts will keep occurring until the job eventually happens to become a short job. In order to avoid this bias, a parameter RepeatOnRestart is included for the Rule Manager (see Table II). If RepeatOnRestart is set to 1.0, then the entire sequence of tasks of an aborted transaction will be repeated on restart. RepeatOnRestart settings are allowed to range from 0 to 1 , thus representing varying likelihoods that a restarted transaction will go through the exact same sequence of tasks that the aborted transaction did.

\section{The Source Module}

The applications of active DBMS's suggest that an open queueing model be used: e.g., in a large-scale control appli-
TABLE III

External Workload Model Parameters

\begin{tabular}{lc}
\hline \multicolumn{1}{c}{ Parameter } & \multicolumn{1}{c}{ Meaning } \\
\hline ArrRate & $\begin{array}{c}\text { Overall Arrival Pattern Parameters } \\
\text { Mean exponential arrival rate of } \\
\text { External Tasks } \\
\text { NumClasses }\end{array}$ \\
Number of classes of External Tasks \\
ClassProb & Per-Class Parameters \\
FileCount & Probability of this class \\
FileProb $i$ & Number of files accessed \\
NumPages & Access probability for file $i$ \\
WriteProb $i$ & Average number of file $i$ pages read \\
PageCPU & Write probability for file $i$ pages \\
WriteCPUFraction & CPU time for processing a page of data \\
& Fraction of read CPU time required to \\
& process a write
\end{tabular}

cation, ET's may arrive at the DBMS in the form of data gathered by a large number of sensors. The Source module is the component responsible for modeling the external workload for the DBMS (i.e., it generates the External Task of each job). Each External Task is sent to the Transaction Manager in the form of an initial transaction.

Table III summarizes the key parameters of the external workload model. Jobs arrive at the system in the form of a Poisson process with arrival rate ArrRate. ${ }^{2}$ The workload model used by the Source characterizes External Tasks into classes, just as RMT's are classified at the Rule Manager. NumClasses specifies the number of different classes of External Tasks. The remaining parameters are similar to those used to model the RMT workload, as described in Section IV-B2.

\section{The Transaction Manager Module}

The Transaction Manager is responsible for accepting transactions from the Source as well as the Rule Manager and modeling their execution. A read access by a transaction begins with a concurrency control request to get access permission. Once the access is granted, a read request for the page is sent to the Resource Manager. If the data page is found in the main memory buffers, no $\mathrm{I} / \mathrm{O}$ is required; otherwise, a disk $\mathrm{I} / \mathrm{O}$ to read the page (possibly accompanied by the flushing of a dirty buffer page) is scheduled. Once the data page is available in the buffer pool, a period of CPU usage follows for processing the page. Write requests are handled similarly.

The input parameters of the Transaction Manager module are listed in Table IV. In addition to modeling the execution of transactions, the Transaction Manager controls the load of the system by limiting the number of concurrently active jobs via the parameter MaxActiveJobs. If a new job is received by the Transaction Manager when there are already MaxActiveJobs jobs in the system, the new job is blocked until one of the active jobs completes execution. Load control at the job level rather than at the transaction level is appropriate for an active system, as the amount of "load" represented by a transaction depends upon the coupling modes employed to couple the job's tasks. Since the overhead associated with transaction

${ }^{2}$ Our model is actually somewhat more general than indicated here; it supports multiple arrival processes, and these can each be either exponential or periodic. 
TABLE IV

Remaining Parameters of tHe Model

\begin{tabular}{|c|c|}
\hline Parameter & Meaning \\
\hline & Transaction Manager Parameters \\
\hline MaxActiveJobs & Maximum number of concurrently running jobs \\
\hline LoggingDelay & $\begin{array}{l}\text { Per-transaction delay caused by flushing a commit } \\
\text { record } \\
\text { Resource Manager Parameters }\end{array}$ \\
\hline NumDisks & Number of disks \\
\hline MinDiskTime & Minimum disk access time \\
\hline MaxDiskTime & Maximum disk access time \\
\hline BufferPoolSize & $\begin{array}{l}\text { Number of page frames in the buffer pool } \\
\text { Concurrency Control Manager Parameters }\end{array}$ \\
\hline CCReqCPU & $\begin{array}{l}\mathrm{CPU} \text { time required to process a concurrency control } \\
\text { request }\end{array}$ \\
\hline
\end{tabular}

startup (which corresponds to the startup time for a lightweight process) is typically on the order of a fraction of a millisecond [21], [11], this cost is not included in the model. However, a delay modeled by LoggingDelay represents the time required to flush $\log$ records at the end of each transaction. Finally, the Transaction Manager also handles transaction commits and aborts.

\section{E. The Resource Manager Module}

The Resource Manager controls the physical resources of the DBMS, including the CPU, the disks, and the buffer pool in main memory. It provides services to both the Transaction Manager and the Concurrency Control Manager. The parameters of the Resource Manager are summarized in Table IV. The DBMS has NumDisks disks plus one CPU. The CPU service discipline is processor sharing. Each of the disks has its own queue, which it serves in a FIFO manner; the Resource Manager assigns a disk to serve a new request randomly, with all disks being equally probable, so the I/O model assumes that the files stored in the DBMS are evenly balanced across the disks. Access times for the disks are uniform over the range [MinDiskTime, MaxDiskTime].

The buffer pool model consists of a set of BufferPoolSize page frames. Each page frame has a "dirty" flag associated with it to indicate whether its data have been updated since it was read into main memory. If a request to read a page is received from the transaction manager, the buffer manager checks whether the requested page is in the buffer pool. If it is present, no disk access is required. If the page is not found, the buffer manager searches for the least-recently-used nondirty page which can be replaced. If such a clean page is available, a disk $\mathrm{I} / \mathrm{O}$ is scheduled to read the requested page into the buffer frame occupied by the replacement victim. If no clean page is found, a disk write is scheduled to write back the least-recently-used dirty page in addition to the read that is scheduled for the requested page. In either case, after the page is read into the buffer pool, the read request is processed by the CPU.

\section{F. The Concurrency Control Manager Module}

The Concurrency Control Manager captures the semantics of the concurrency control algorithm of choice. As was illustrated in Fig. 1, the Concurrency Control Manager is responsible for handling concurrency control requests made by the Transaction Manager. These include read and write access requests, requests to get permission to commit a transaction, and several types of concurrency control management requests to initialize and terminate transactions. The Concurrency Control Manager has a variable number of parameters. One parameter, CCReqCPU, specifies the amount of CPU time iequired to process a read or write access request; this parameter is present for all algorithms. Additional parameters can be introduced on a per-algorithm basis as needed.

For the experiments described in this paper, a variation of the Wound-Wait (WW) algorithm described in [26] is used for concurrency control. We chose this algorithm because it is relatively easy to model, and its timestamp-based conflict resolution strategy makes it possible to introduce an interesting modification to the algorithm, as discussed below. Also, the WW algorithm can be modified later to allow priorities to be associated with transactions in order to model time constraints, as proposed in [1], [2], [29], and [5].

In the WW algorithm, locks are acquired and released in a two-phase fashion [14]. Deadlock is prevented via the use of timestamps rather than by maintaining waits-for information and then checking for deadlocks. In the original WW algorithm, each transaction is numbered according to its initial startup time, and younger transactions are prevented from making older ones wait. If an older transaction requests a lock held by a younger transaction, which would lead to the older transaction waiting, the younger transaction is "wounded," i.e., it is restarted. Younger transactions are permitted to wait for older transactions, however. Deadlocks are impossible because any cycle of waiting transactions would have to include at least one instance where an older transaction is waiting for a younger one that is blocked as well, and this is not allowed.

In order to model the relationship between the transactions that make up a job, the following variation was introduced in the WW timestamp allocation scheme. Let $J o b_{i}$ consist of transactions $T_{i 1}, \cdots, T_{i m}$, and let $J o b_{j}$ consist of transactions $T_{j 1}, \cdots, T_{j n}$. If $J o b_{i}$ starts before $J o b_{j}$, then all of $J o b_{i}$ 's transactions $T_{i k}, k=1, \cdots, m$, will have earlier timestamps than any of the transactions of $J o b_{j}$. Intuitively, the purpose of this modification is to favor tasks of jobs that arrive early over those of later arrivals, thus introducing an element of job-based priority to the concurrency control algorithm. As a result of this modification, tasks of older jobs are more likely to obtain locks and less likely to be aborted than tasks of younger jobs. (Note that this timestamp allocation scheme is independent of both the type of coupling between the tasks and the time ordering of the individual tasks of different jobs.)

\section{EXPERIMENTS AND RESULTS}

In this section, we present performance results for the four types of RMT coupling described in Section III under various assumptions about the database size, the number of data pages accessed by each task of a job, the number of tasks per job, and the extent of data sharing between the tasks of a job. As stated in Section I, it is obvious that in the absence of 
significant overheads associated with transaction startup and commit, breaking a job into many transactions should improve performance by lessening data contention. The experiments in this section were designed with two goals in mind: to provide quantitative information about the extent to which performance improves as a consequence of job decoupling, and to provide insights into the performance tradeoffs between the various coupling modes. Our performance results are preceded by a discussion of the performance metrics of interest and the parameter settings used to obtain the results presented here.

\section{A. System Performance Metrics}

As stated earlier, job response time is a measure of the speed with which the DBMS reacts to external stimuli. In order to focus on the performance impact of using rule semantics for transaction coupling, we examine the average job response time as a function of the job arrival rate for each of the four types of coupling modes. Job response time is computed by subtracting the startup time of the ET of the job from the time at which its last RMT finally completes successfully. Each experiment was run until a total of approximately 4500 jobs completed in order to obtain a statistically significant sample of job executions.

\section{B. Parameter Settings}

Since general-purpose active DBMS's are not a commercial reality yet, there is no data available regarding "typical" workload characteristics such as the likelihood of rules being fired in such systems. Furthermore, we are concerned more here with performance trends than with exact performance predictions. Consequently, we have run simulations with a variety of settings for the triggering probability parameters (ExtCheckProb, ActionProb, and ActCheckProb) of our simulation model. In this paper, we present results that are representative of the overall trends we observed as we varied the workload parameters of the model.

Table $\mathrm{V}$ gives the values of the key simulation parameters in our base experiment. As subsequent experiments are discussed, we describe the variations in the parameters for each experiment. The database is modeled simply as a single file consisting of 500 data pages. Jobs arrive at the system in a single exponential stream; the job arrival rate is varied in each experiment. Each task accesses five pages of data on the average. External Tasks and Actions update each page that they access with a probability of $1 / 3$, while Checkers are readonly tasks. It takes an average of $8 \mathrm{~ms}$ of CPU time to process each data page that is read, and an average of 2 additional milliseconds to process each update. The maximum number of jobs that may run concurrently is set to 15 . There are two disks available for $\mathrm{I} / \mathrm{O}$, and each disk has an average access time of $20 \mathrm{~ms}$. The buffer pool can hold up to 50 pages in memory at a time. The concurrency control CPU overhead is assumed to be negligible compared to the CPU time required for page processing. A commit record logging delay of $10 \mathrm{~ms}$ is added to each transaction at commit time. The probability that an External Task (or an Action) is followed by a Checker is set at $2 / 3$, and the probability that a Checker is followed
TABLE $\mathrm{V}$

Base Parameter Settings

\begin{tabular}{ll}
\hline \multicolumn{1}{c}{ Parameter } & \multicolumn{1}{c}{ Base Setting } \\
\hline NumFiles & 1 \\
FileSize & 500 pages \\
ArrRate & varied from 0.2 jobs/second to 8 jobs/sec \\
NumClasses & 1 \\
NumPages & 5 \\
WriteProb & $1 / 3$ \\
PageCPU & $8 \mathrm{~ms}$ (for reads); $2 \mathrm{~ms}$ (for writes) \\
MaxActiveJobs & 15 \\
LoggingDelay & $10 \mathrm{~ms}$ \\
NumDisks & 2 \\
MinDiskTime & $10 \mathrm{~ms}$ \\
MaxDiskTime & $30 \mathrm{~ms}$ \\
BufferPoolSize & $50 \mathrm{page}$ frames \\
CCReqCPU & $0 \mathrm{~ms}$ \\
ExtCheckProb & $2 / 3$ \\
ActionProb & $1 / 2$ \\
ActCheckProb & $2 / 3$ \\
RNumPages & 5 \\
RWriteProb & 0 (for Checkers); $1 / 3$ (for Actions) \\
RPageCPU & $8 \mathrm{~ms}$ (for reads); 2 ms (for writes) \\
ShareMode & No Sharing \\
ShareProb & 0.0 \\
RepeatOnRestart & 1.0 \\
\hline
\end{tabular}

by an Action is set at $1 / 2$. Thus, approximately one third of all jobs consists of just one task, another third consists of two tasks, and the remainder consists of more than two tasks; the mean number of tasks per job works out to be 2.33. Data are not shared between the adjacent tasks of a job. Finally, the restart parameter is set so that an aborted transaction always repeats its original sequence of tasks.

In order to isolate the effects of using each of the four coupling options, each simulation had only one type of coupling between the tasks of a job. For each arrival rate, four simulations were run: one in which all jobs consisted of S-coupled tasks, one in which only EC-coupling was allowed, one in which only CA-coupling was allowed, and one in which only $\mathrm{N}$-coupling was allowed. In addition to these four simulations, we ran one simulation for each arrival rate to obtain the average job response time in the absence of concurrency control conflicts in order to provide a performance bound against which to compare the various coupling modes. In each of our graphs, a curve labeled No-CC shows the results of this simulation.

\section{The Base Experiment}

Fig. 2 shows the average job response times for the parameter settings in Table V. At low arrival rates, the response time of jobs does not vary significantly with the type of coupling. As the arrival rate is increased, all of the curves begin to move upwards. The S-coupling curve separates from the others first, while the EC-coupling, CA-coupling, N-coupling, and No-CC curves remain fairly close to one another for most arrival rates. Furthermore, the average job response times for EC-coupled jobs are consistently higher than the average job response times for CA-coupled jobs. This is an interesting result, as it shows that transaction size is not the only factor affecting 


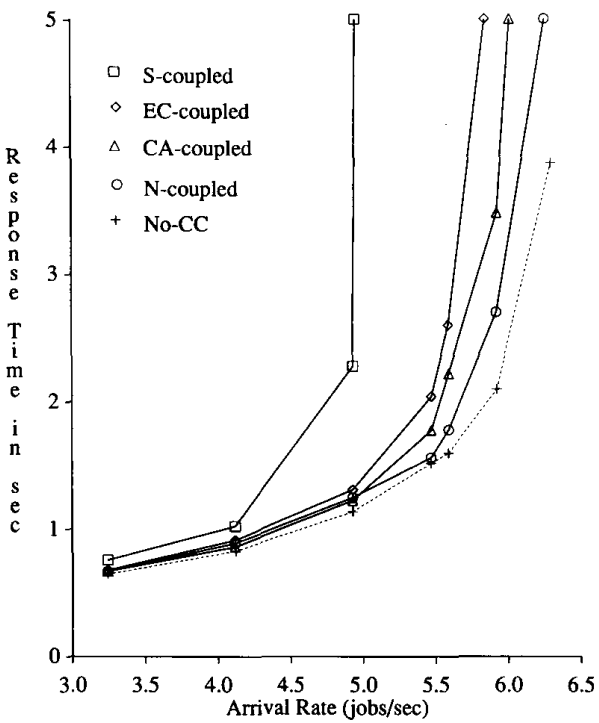

Fig. 2. Database size $=500$. (TaskSizes $=[5,5,5]$, no sharing).

the performance of the system; the average number of pages accessed per transaction when EC-coupling is used is close to that when CA-coupling is used, since both schemes combine two successive tasks into a single transaction. As in any open system, job response time grows dramatically when the job arrival rate is increased beyond a certain threshold value for each type of coupling. This rise in job response time is so dramatic that some of the higher response time values do not fit into the graph and are represented (in all experiments) by extrapolated lines at the right end of each curve. Fig. 2 shows that the threshold at which this phenomenon occurs is reached earliest by the S-coupled jobs. EC-coupled jobs reach the point of saturation next, and CA-coupled jobs reach their threshold just before N-coupled jobs.

The elements contributing to the average response time of a job are the costs of reading and updating pages and the costs of resolving concurrency control conflicts. As explained in Section IV-D, CPU costs for transaction startup are assumed to be negligible, and transaction commit costs (in the form of the $10 \mathrm{~ms}$ logging delay) are small relative to the overall response times observed. The number of pages read and written by a given job does not vary with the type of coupling used in the system (in the absence of restarts), so the differences in job response times for the four types of coupling are almost entirely due to concurrency control costs. In order to understand how concurrency control affects performance, we must examine two factors: blocking delays and restart rates. A quantitative display of these factors is given in Figs. 3 and 4. The average total amount of time a job has to spend waiting for locks is presented in Fig. 3, whereas the average number of times that a transaction is restarted is presented in Fig. 4. Both figures indicate that the manner in which tasks are coupled to form transactions has a dramatic impact on the amount of time that jobs spend redoing work (due to restarts) or waiting for locks to be granted.

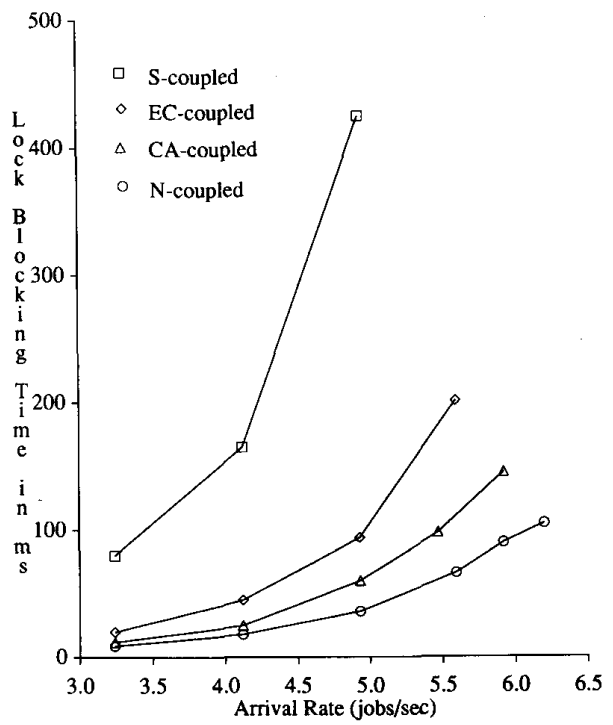

Fig. 3. Lock blocking time per job, DBSize $=500$. (TaskSizes $=[5,5,5]$, no sharing).

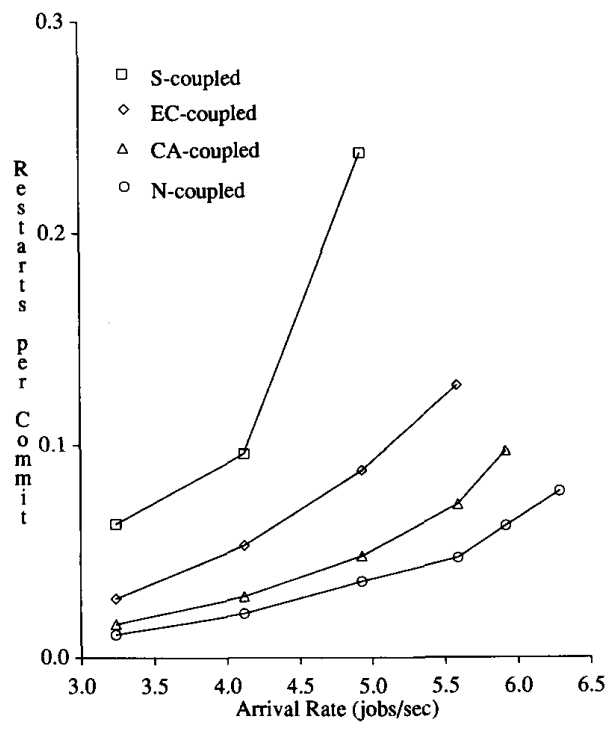

Fig. 4. Restarts/commit, DBSize $=500$. (TaskSizes $=[5,5,5]$, no sharing).

In order to understand just how the locking behavior of jobs depends on their coupling mode, consider a job that consists of three tasks: an ET, a Checker, and an Action. When the job is S-coupled, it holds all of its locks until the Action task is completed. With EC-coupling, the job holds its locks until the end of the Checker task, releasing them before the Action task begins. A CA-coupled job releases its locks immediately after the completion of the External Task. Such a job holds the Checker locks (which are all read locks) and the Action locks until it commits. When the job is $\mathrm{N}$-coupled, it releases locks when each task is completed. Two issues related to locking affect the average blocking time of the four coupling 
alternatives. The first is the average duration for which locks are held by a transaction. Obviously, the longer a transaction holds on to its locks, the longer other transactions will have to wait to lock a data item. The second issue is the type of lock held. Since External Tasks consist of updates as well as reads, both S-coupled and EC-coupled jobs hold write locks for long durations, while CA-coupled jobs hold only read locks for long durations. Since read locks can be shared, while write locks cannot, holding read locks for longer durations does not have as much of a negative impact on job response time as holding write locks. This explains the differences in the average blocking times of EC-coupled and CA-coupled jobs in Fig. 3. Although both modes lead to the same average transaction size here, EC-coupled jobs hold write locks longer and thus cause longer blocking delays; this explains why average transaction size alone does not determine job response time. When write locks are held for long durations, as in ECcoupling or S-coupling, increased data contention results in a significant increase in job response time. S-coupling has the longest blocking time because all of the locks acquired by a job are held until the very last task of the job completes execution.

Another factor related to the performance impact of concurrency control is transaction restarts. Restarts increase resource utilization and lengthen the execution path of transactions. Fig. 4 shows that the average number of restarts per commit is fairly low in this experiment. In most cases, there is significantly less than one restart per ten commits. Recall also that our Wound-Wait based concurrency control algorithm tends to restart younger tasks more often than it restarts older tasks. These two facts lead us to expect that the increase in resource utilization due to restarts will not be significant - and our measurements show that this increase is indeed small (less than $3 \%$ in most cases). However, the increase in the average number of locks that must be acquired or reacquired by a transaction has a major impact on job response time. Our measurements show that a significant proportion of the time "wasted" due to restarts was spent waiting again for locks rather than utilizing the physical resources of the system. Finally, it is also important to note that restarted transactions represent a different amount of "wasted" time for each type of coupling. In the case of S-coupling, restarting a transaction is equivalent to restarting an entire job, since each job consists of only one transaction. In contrast, in the case of $\mathrm{N}$-coupling, the amount of time wasted by a restart is bounded by the time taken by a single task of a job.

So far we have focused on how job response time is affected by the different coupling modes. Another interesting performance measure is the average time that users must wait for their tasks to complete. This metric (called the ET response time of the system) is presented in Fig. 5 for the four coupling modes. This measure represents the user's point of view of the performance of the active DBMS, whereas the job response time represents the system administrator's point of view. For the entire range of arrival rates, as expected, coupling RMT's with ET's causes a significant increase in the response time observed by a user. More significantly, all of the less strict coupling modes offer major performance improvements over

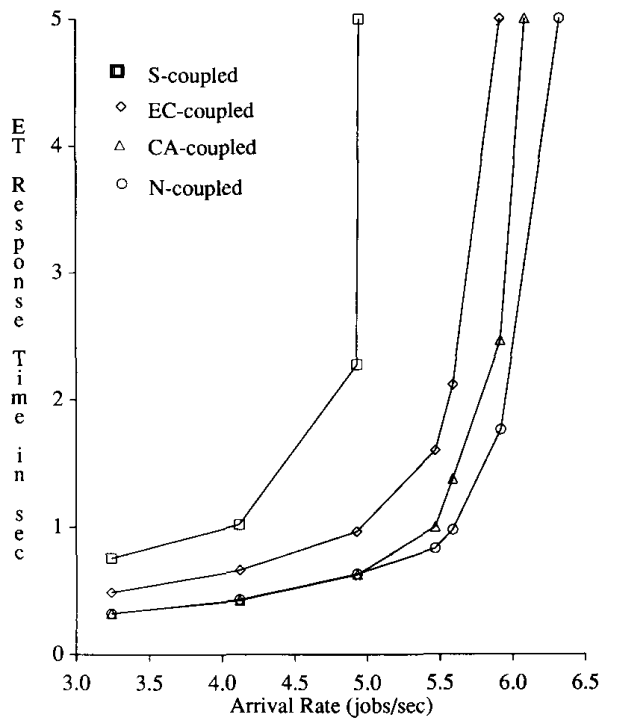

Fig. 5. ET response time, DBSize $=500 . \quad($ TaskSizes $=[5,5,5]$, no sharing).

S-coupling, including EC-coupling (even though it does not completely decouple the ET from the RMT's).

To summarize, the results of the base experiment clearly demonstrate that the way in which transaction boundaries are set in an active DBMS can have a very significant impact on both the quality of service experienced by its users and the location of the saturation point of the system. The results also indicate that the key factor that influences job response time with the parameter settings of the base experiment is data contention rather than resource contention. As one would expect, jobs that were S-coupled performed significantly worse than jobs that did not couple all of their rule management activity to the External Task. The results also show that while jobs that were completely decoupled performed the best of all, jobs whose Checkers were coupled to their Actions (i.e., where CA-coupling was used) performed almost as well over a wide range of arrival rates. This is a reassuring result, for as shown in the example in the Appendix, CA-coupling seems likely to be well-suited to several active DBMS applications. ${ }^{3}$ It is important to note that from a performance point of view, augmenting user transactions with code to check conditions (e.g., violations of integrity constraints) and to invoke actions in response to satisfied conditions is basically the same as S-coupling. We can thus conclude from the results of the base experiment that not only can active DBMS's simplify the task of application programming, but they can also lead to performance gains.

\section{Experiment 1: The Effect of Data Contention}

In this experiment, we examine job response times for the

\footnotetext{
${ }^{3}$ In particular, CA-coupling provides the guarantees that i) if an event occurs, the corresponding condition will eventually be checked, and ii) any action will be executed in the same transaction-consistent state in which its condition is determined to be true.
} 


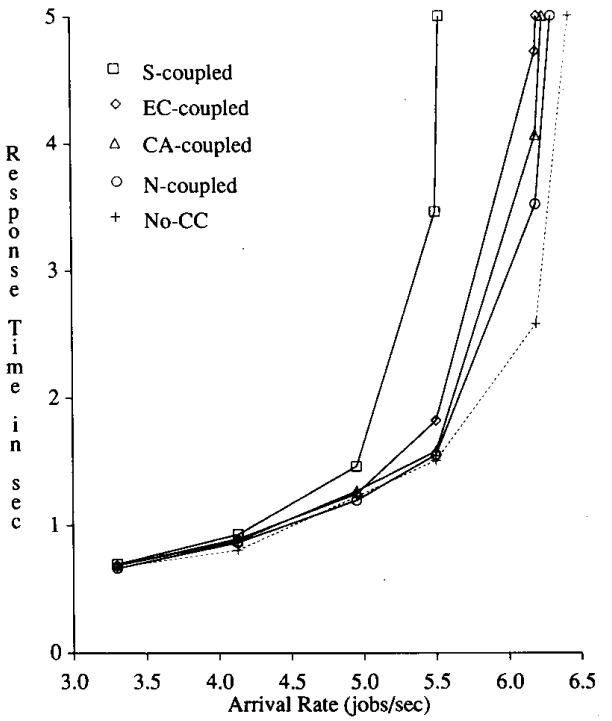

Fig. 6. Database size $=750$. (TaskSizes $=[5,5,5]$, no sharing).

four coupling types as the size of the database is changed. ${ }^{4}$ Three database sizes $(750,500$, and 250 pages) are studied, and the buffer pool size is kept at $10 \%$ of the database size in each case. Each task of a job accesses 5 pages on the average, as in the base experiment.

Fig. 6 shows the average job response times for a database consisting of 750 pages, where the buffer pool can accommodate 75 such pages. Recall that Fig. 2 presented the response time results when the database size is set at 500 pages and the buffer pool size is 50 pages. In Fig. 7, the job response times for a database size of 250 pages and a buffer pool size of 25 pages are shown. In Fig. 8, we compare the job response times for an arrival rate of 5 jobs/second for the three different database sizes. Since the system becomes unstable for S-coupled jobs at an arrival rate close to 4 jobs/second in the 250-page case, we ran further experiments with database sizes of between 500 and 250 pages specifically in order to obtain the third point for the S-coupled case in Fig. 8 .

In Fig. 6, where the database size is 750 pages, the response time curves for the four types of coupling remain close to each other for most arrival rates, and the system remains stable for arrival rates as high as 6 jobs/second. As the size of the database is decreased to 500 pages in Fig. 2 and then to 250 pages in Fig. 7, the curves for S-coupled and EC-coupled jobs move away from the other curves at lower arrival rates than before. The separation between these curves and the curves for CA-coupling and $\mathrm{N}$-coupling also grows, and increased data contention causes the system to become unstable more rapidly with increasing arrival rates. In particular, S-coupled jobs make the system unstable at arrival rates of less than 4 jobs/second when the database contains 250 pages, but when the database size is 750 pages, $50 \%$ higher arrival rates can

\footnotetext{
${ }^{4}$ Varying the database size in this manner can be thought of as a way to vary the extent to which "hot spots" exist in a larger database, as shown in [34].
}

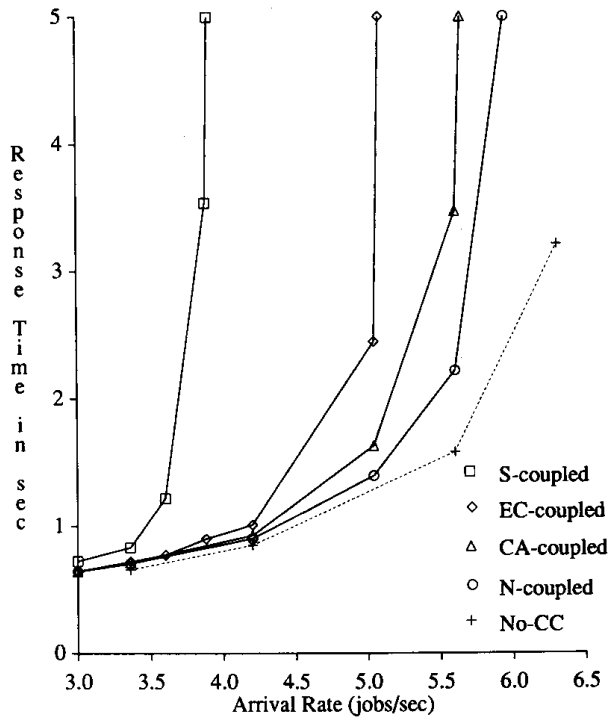

Fig. 7. Database size $=250$. (TaskSizes $=[5,5,5]$, no sharing).

be sustained by the system without losing its stability for S-coupled jobs. The reduction in the size of the buffer pool from Fig. 6 to Fig. 2 and again from Fig. 2 to Fig. 7 may at first seem likely to contribute to the positioning of the response time curves in the three cases. However, a closer examination reveals that the No-CC curves in the three figures coincide almost exactly for all but the highest arrival rates shown. Even at arrival rates (between 6 and 6.5 jobs/second) that cause the system to enter a state of saturation when concurrency control is in use, the spread between the No-CC curves in the three figures is less than $10 \%$ of the minimum response time. Thus, changing the buffer pool size does not bias the results of this experiment significantly, and buffer hits may be ignored as a factor in comparing the three sets of curves.

Fig. 8 illustrates the increasingly important impact of task coupling semantics on system performance as data contention increases. For low levels of data contention, the differences in performance between the different types of coupling are relatively small. As data contention increases, however, N-coupling leads to slightly better performance than CA-coupling, while CA-coupling performs better than EC-coupling. As expected, S-coupling results in the worst performance. Note that CA-coupling and $\mathrm{N}$-coupling again provide similar levels of performance. Since a temporary period of high data contention can easily occur even in a passive DBMS (e.g., when there is a "hot spot" in the data), it should be clear that it is important for performance that the weakest type of coupling permitted by each rule's semantics be selected and used in the rule's definition.

\section{E. Experiment 2: The Effect of Task Complexity}

Tasks associated with jobs in active database systems will have application-dependent data access characteristics. External Tasks, Checkers, and Actions may vary in the relative amounts of data that they need to access. Also, their data 


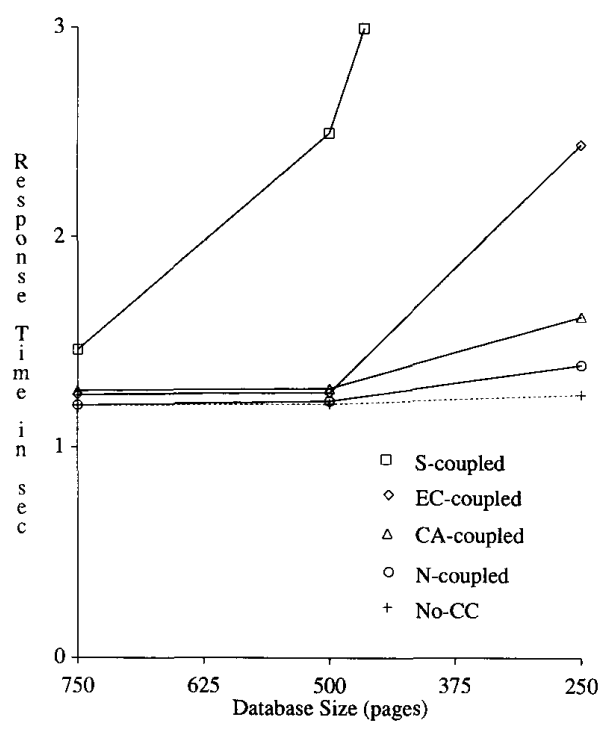

Fig. 8. Arrival rate $=5$ jobs $/$ second. (TaskSizes $=[5,5,5]$, no sharing).

access characteristics will be influenced by the degree to which the physical design of the passive database (e.g., the indexes provided) supports the queries involved in the rules. In order to study the performance impact of variations in task size, simulations with various combinations of task sizes were run; results for three combinations are presented here. All of these experiments were conducted on a 500-page database.

Recall that in the base experiment, all of the tasks of each job access 5 pages each, on the average. This set of parameters is abbreviated SSS (for Short External Tasks, Short Checkers, and Short Actions). Fig. 2 showed the average response time of jobs with the SSS task pattern. The second pattern of tasks studied was SLS (Short External Tasks, Long Checkers, and Short Actions). External Tasks and Actions again access 5 pages each, while Checkers now access 20 pages each on the average. Fig. 9 shows the response times of jobs with SLS task patterns. Fig. 10 presents job response times for jobs having an SLL task pattern - where External Tasks are short (5 pages each) while both Checkers and Actions are long (20 pages each). Fig. 11 shows the maximum sustainable arrival rates at which the system remains stable for the SSS, SLS, and SLL task patterns.

Figs. 2, 9, and 10 show that significant performance changes occur when the task access patterns are changed. In Fig. 2, all of the curves except for the S-coupled one are quite close to one another. When the size of Checkers increases, in Fig. 9, the differences between the performance of the four coupling types become much more pronounced. The slopes of the curves increase more rapidly, and the maximum sustainable arrival rates become smaller. The difference between the No$\mathrm{CC}$ curve and the $\mathrm{N}$-coupled curve is still relatively minor here, though. When both Checkers and Actions are large, as in Fig. 10, the response time curves become very steep even at low arrival rates. An interesting phenomenon in the SLL case is that, for the first time, CA-coupling performs worse than

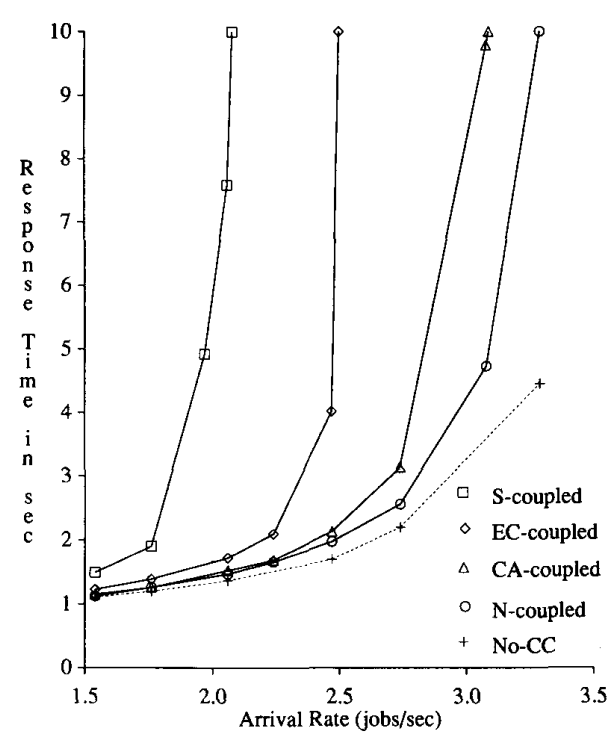

Fig. 9. S-L-S task pattern. (TaskSizes $=[5,20,5]$, DBSize $=500)$.

EC-coupling. The reason for this is that, in this case, External Tasks are short whereas Actions are long. Thus, if we consider jobs having three tasks or less (which constitute most of the jobs in the simulation), EC-coupling involves holding locks for a considerably shorter time than CA-coupling. Another feature of the SLL case is that even the $\mathrm{N}$-coupled curve is quite far from the No-CC curve, indicating that the increased sizes of Checkers and Actions cause significant concurrency control conflicts even when job tasks are uncoupled. Fig. 11 reveals that, as RMT's become more complex, the relative impact of the coupling mode on performance increases. For example, the ratio of the maximum sustainable arrival rate for $\mathrm{N}$-coupled jobs to the maximum sustainable rate for S-coupled jobs goes up from 1.25 in the SSS case to 1.75 in the SLL case. These results indicate that system performance can be affected significantly by both the design of the RMT's of a rule (i.e., the relative complexity of Checkers and Actions) and the degree to which their data access needs are supported by the physical organization of the passive database.

\section{F. Experiment 3: The Effect of Cascading Triggers}

In Experiment 3, the probabilities of executing both Checkers and Actions were increased to study the effect of increased job size (in terms of the number of tasks per job) on system performance. A number of probability combinations were tried; here we present results for one set of probabilities, where the mean number of tasks per job is about twice the number in the base experiment. By increasing CheckProb from $2 / 3$ to $4 / 5$ and ActionProb from $1 / 2$ to $4 / 5$, the mean number of tasks per job was increased from 2.33 to 5 . (Note that the transaction boundaries imposed by the different coupling semantics on a job consisting of exactly five tasks were shown in Table I in Section III.) The database size was kept fixed at 500 pages. Recall that response times for jobs with SSS and SLS task patterns, with an average of 2.33 tasks per job in 


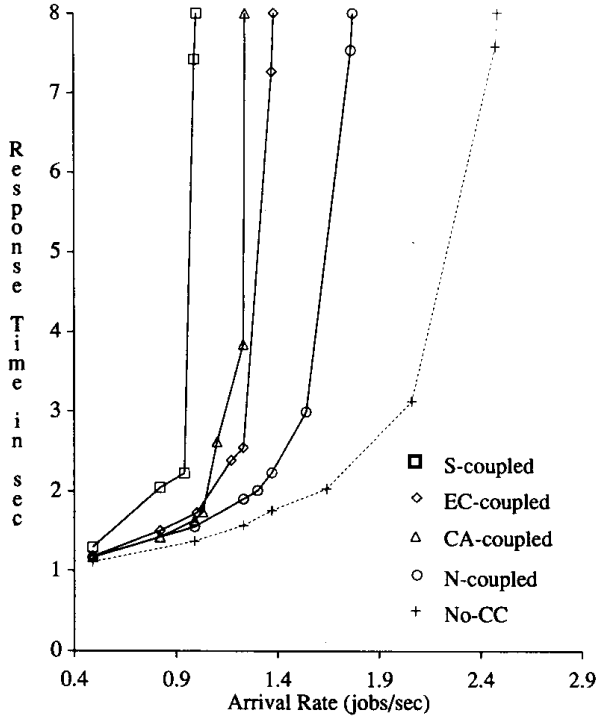

Fig. 10. S-L-L task pattern. (TaskSizes $=[5,20,20]$, DBSize $=500)$.

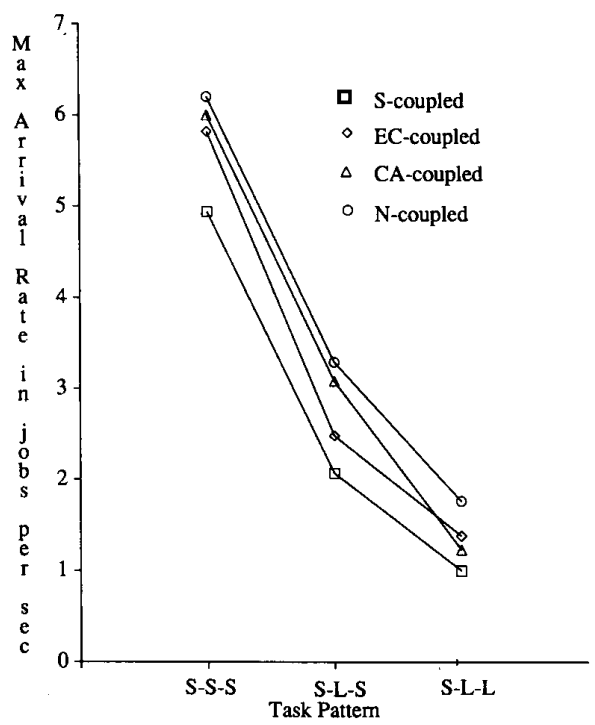

Fig. 11. Maximum sustainable arrival rates. (DBSize $=500)$.

each case, were shown in Figs. 2 and 9, respectively. Fig. 12 shows the job response times of SSS pattern jobs when jobs have 5 tasks on the average. In Fig. 13, jobs again have 5 tasks, and the task pattern in now SLS.

The results of this experiment show the degree to which decoupling the tasks of a job (i.e., avoiding S-coupling) becomes increasingly important as the job size increases. As in earlier experiments, S-coupling performs worst of all; the significant feature of Figs. 12 and 13 is that the curves for the less strict coupling modes remain relatively close to each other (and far from the S-coupled curve). The reason that ECcoupling and CA-coupling provide very similar performance in Fig. 12 is that both of these coupling modes involve releasing

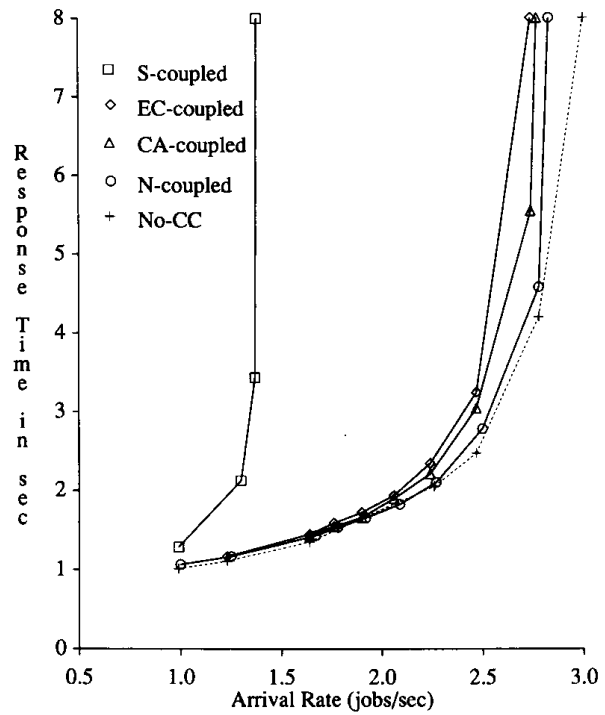

Fig. 12. Mean number of tasks per job $=5.0$. (TaskSizes $=[5,5,5]$, DBSize $=500$ ).

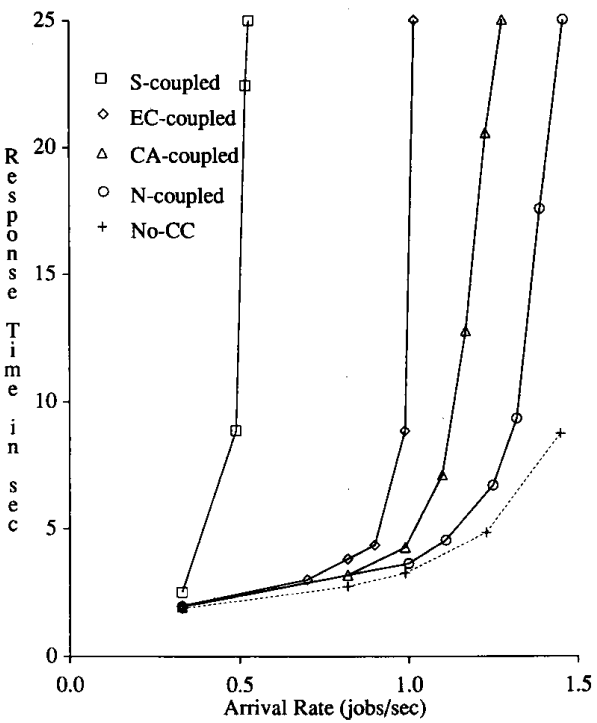

Fig. 13. Mean number of tasks per Job $=5.0$. (TaskSizes $=[5,20,5]$, DBSize $=500$ )

locks after alternate tasks of a job. When the tasks are short and there are many tasks per job, the effect of the type of lock (read versus write) that is held longer is diluted to some extent.

\section{G. Experiment 4: The Effect of Data Sharing Between Tasks}

As discussed earlier, it is likely that there will be some degree of data sharing between successive pairs of tasks of a "real" job. In Experiment 4, the extent of data sharing between successive tasks is varied. The results of the base experiment, in which there was no enforced data sharing between tasks, were shown in Fig. 2. (Since data pages are chosen randomly 


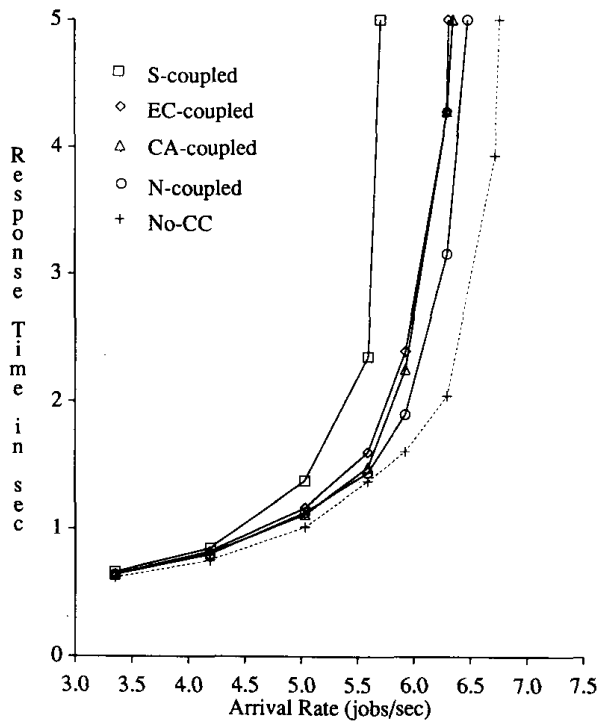

Fig. 14. 50\% data sharing. (TaskSizes $=[5,5,5]$, DBSize $=500$ ).

from the database, there may be some inherent sharing of data between tasks even in this case, but it is rare enough to be ignored.) Figs. 14 and 15 show the results of experiments with Read-Read sharing, where the read-set of each task of a job overlaps with the read-set of the next task of the same job. Three levels of data sharing are studied: no sharing (which corresponds to the base experiment), 50\% sharing, and $100 \%$ sharing. Recall that the level of sharing represents the probability that any given page will be accessed by two consecutive job tasks, subject to the constraints of the relative size of the tasks. (While the average size of the tasks is 5 pages each, the actual size of different tasks may vary between 3 and 7 pages. Thus, if task $T_{i}$ of a job accesses 3 pages and its successor task $T_{i+1}$ accesses 7 pages, a maximum of 3 pages can be shared between the two tasks.) Fig. 16 shows the maximum arrival rates that the system can support for an average job response time of $2 \mathrm{~s}$ for the four different types of coupling as the level of sharing is changed. (The points on Fig. 16 were interpolated from the data in Figs. 2, 14, and 15.)

When there is no data sharing between job tasks, all the curves (except the curve for S-coupling) remain fairly close to one another, as seen in Fig. 2. As the level of sharing is increased from 0 to $50 \%$, the curves for EC-coupling and CAcoupling move still closer, almost merging with each other. The S-coupling curve also moves somewhat closer to the other three, but S-coupled jobs still perform noticeably worse than the other types of coupling. Fig. 15 shows that when all of a job's tasks access essentially the same data, the performance of all four types of coupling becomes very similar. All four curves remain close together for the entire range of sustainable arrival rates. Fig. 16 shows that at low levels of data sharing, the maximum job arrival rate that the system can support in order to provide a given average response time ( $2 \mathrm{~s}$ in this case) varies significantly with task coupling semantics. At high levels of data sharing, however, this difference becomes negligible.

Data sharing affects job response time in two ways. If data

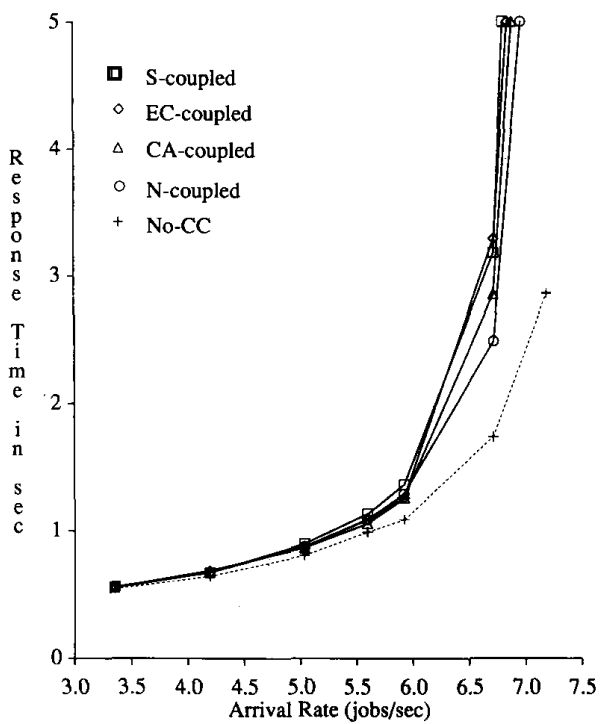

Fig. 15. $100 \%$ data sharing. (TaskSizes $=[5,5,5]$, DBSize $=500$ ).

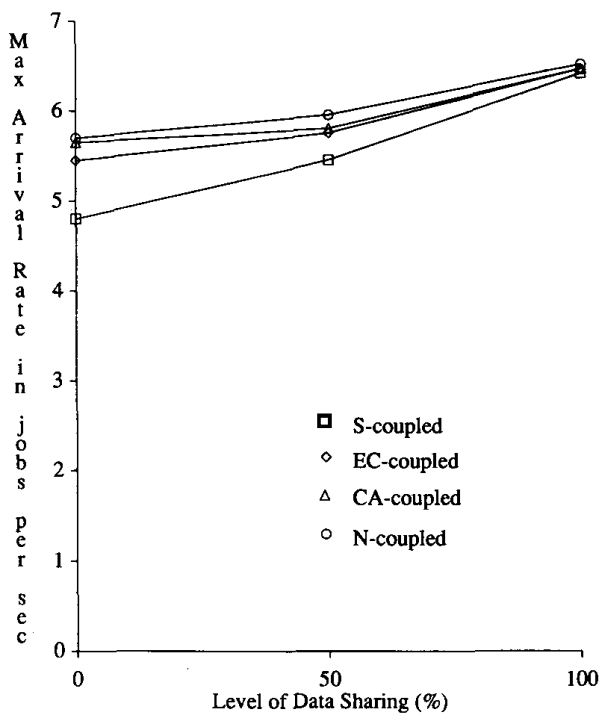

Fig. 16. Job Response Time $=2 \mathrm{~s}$. $($ TaskSizes $=[5,5,5]$, DBSize $=500)$.

are shared between successive tasks, more buffer pool hits are likely. Also, fewer locks need to be obtained in the Scoupling, EC-coupling, and CA-coupling cases, as the locks acquired in one task of a job within a transaction do not need to be reacquired in the succeeding phase. An examination of the results for buffer pool hit ratios, disk utilization, and number of locks avoided by data sharing (not shown here) indicate that, while both buffering and locking factors affect the results, the relative performance of the four types of coupling is affected most by the fact that fewer locks are required (except for $\mathrm{N}$-coupled jobs) as data sharing is increased. In $\mathrm{N}$-coupling, locks are released at the end of every task, so the amount of locking activity is unaffected by data sharing; this is why $\mathrm{N}$-coupling does not show as much improvement as the other 
types of coupling when the amount of data sharing is increased. The main point illustrated by this experiment is that coupling semantics affect performance significantly even when half the data of each task is shared with the subsequent task. It is only when the ET and the RMT's of a job share data to a very large extent that the coupling semantics lose their relevance to system performance.

\section{CONCLUSIONS AND FUTURE WORK}

The workload of an active DBMS consists of two types of activities: External Tasks, which are the tasks submitted by users or application programs, and Rule Management Tasks, which are caused by the triggering of rules stored in the active database. In this paper, we have described a model of an active DBMS where the triggering of rules is modeled via probabilities. This use of probabilities provides a mechanism for studying the performance of active DBMS's without having to model the details of their rule-base contents. One interesting question that arises in an active DBMS is: How should ET's and RMT's be combined into transactions? Most proposals for integrating rules with database systems give the user little control, if any, over how tasks are combined into transactions. An alternative is to provide users with the capability to exploit rule semantics by specifying where transaction boundaries should be placed, as in HiPAC [16], [10]. In this paper, we have studied the performance impact of using such a capability to determine task coupling in an active DBMS. In particular, we have examined the effects of alternative coupling approaches while varying data contention, task complexity, the number of tasks generated recursively by rules, and the amount of data shared by the tasks of a job.

The experiments described here demonstrated the extent to which the performance of an active DBMS is sensitive to the coupling semantics of rules. We examined four of the coupling modes possible under the Event-Condition-Action rule model [10]: S-coupling, where all the tasks of a job are strictly coupled as a single transaction; EC-coupling, where an External Task is coupled with the RMT's (Checkers) that test for rule satisfaction, but not with the RMT's (Actions) that perform the actions for satisfied rules; CA-coupling, where Checkers and Actions are coupled together, but not with the External Tasks; and N-coupling, where each task is a transaction by itself. We found that, under light loads, the type of coupling did not affect job response time appreciably since there were relatively few concurrency control conflicts. However, we did observe that the coupling mode had a significant impact on the mean response time of External Tasks (i.e., the response time from a user's perspective) under such loads. Throughout the study, the response times of External Tasks that were coupled with Rule Management Tasks (i.e., using S-coupling or EC-coupling) were found to be significantly higher than the response times of External Tasks that were executed as stand-alone transactions (i.e., executed using CA-coupling or $\mathrm{N}$-coupling). ${ }^{5}$

\footnotetext{
${ }^{5}$ Note that user tasks are also decoupled from rule management activity in loosely-coupled knowledge base management systems (where a rule manage polls the DBMS periodically). However, such systems cannot efficiently provide the level of responsiveness possible with an active DBMS [10].
}

As data contention was increased in our experiments (by increasing the arrival rate or the size of jobs, or by decreasing the database size), the impact of the various coupling modes on job response time became apparent. While the average transaction size was a significant factor determining system performance, it was not the only one, as shown by the differences in the performance of EC-coupling and CAcoupling when the mean transaction sizes were equal for the two modes. In general, $\mathrm{N}$-coupled jobs had the best response times, followed closely by CA-coupled jobs, while EC-coupled jobs performed worse but still better than S-coupled jobs. We also found that the size of RMT's may affect the relative behavior of the coupling modes-for example, when Actions and Checkers were significantly larger than External Tasks, CA-coupling performed worse than EC-coupling. Sharing of data between tasks of a job resulted in improved response times, but the coupling mode choice was found to be important except when the degree of data sharing was extremely high.

Two interesting trends were observed in our experiments. The first is that CA-coupled jobs perform almost as well as $\mathrm{N}$-coupled jobs over a wide range of system loads for many of the workloads studied. This is significant because of the guarantees that $\mathrm{CA}$-coupling can provide (see footnote 3 , Section V-C). The second trend that we observed is that the use of any type of task decoupling (EC-coupling, CAcoupling, or $\mathrm{N}$-coupling) results in significant performance gains over S-coupling. That is, the relative performance differences among EC-coupling, CA-coupling, and $\mathrm{N}$-coupling were usually smaller than the relative performance difference between any one of these three and S-coupling. This is significant because the S-coupling results are also indicative of what the response times would be for transactions that have been explicitly augmented with code to check conditions and perform actions, as is often done in systems that do not provide rule support. The experiments that involved changes in RMT complexity and in the number of tasks per job also indicate that rules should be designed with care, as both the distribution of work between Checkers and Actions and the likelihood of triggering recursive RMT's affected performance significantly. In addition, they indicate that the passive database should be designed to support efficient access by the RMT's.

In the study reported here, we have dealt exclusively with transaction management issues in active databases. Our model of an active DBMS provides us with a framework for studying other aspects of active database management as well. We have already studied the problem of scheduling the physical resources (buffers, CPU's, and disks) of a passive DBMS in the presence of priorities [8], [18]. The next focus of our research will be to extend our work in priority scheduling to active databases, where priorities can be used to meet time constraints. The ultimate goal of this research effort is to design a set of scheduling policies for an active DBMS that encompass both transaction scheduling and resource scheduling.

\section{APPENDIX}

A Coupling Mode EXAmple

The purpose of this Appendix is to illustrate how one 
Parts(pno, pname, size, weight, importance)

Stock(pno, quantity, ordered)

Prices(pno, supplier, cost)

Fig. 17. Information about parts in stock.

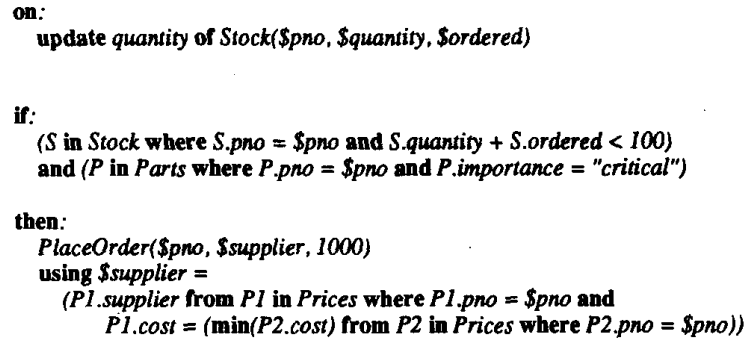

Fig. 18. Inventory control rule for critical parts.

can exploit rule semantics to determine where transaction boundaries should be placed in an active DBMS. In particular, we use an example based on an inventory control rule to show how changes in the semantics of a rule affect the way in which ET's and RMT's can be coupled into transactions. The coupling alternatives discussed here are a subset of the transaction coupling modes that can be specified for rules in the HiPAC system [16].

In this example, we will assume that we have an active DBMS controlling the operation of a completely automated warehouse, with parts being requested at terminals and obtained from the warehouse via a computer-controlled delivery system. Fig. 17 summarizes the part-related information that is kept in the passive portion of the database for this application. The DBMS stores a description of each part, the quantity of each part both in stock and on order, and the current price for each part from each supplier that carries that part. Checkouts from the warehouse are monitored by the inventory control rules presented in Fig. $18 .^{6}$ When a checkout of a critical part reduces the sum of the amount in stock and the amount on order to a level below 100 , the rule places an order for additional 1000 parts from the supplier who is offering the part at the lowest price at the moment. The ordering action itself is performed by a procedure called PlaceOrder, which generates a requisition and also updates the number of part instances on order.

Fig. 18 says nothing about how transaction boundaries should be imposed for the inventory control rule. One possible coupling mode in the HiPAC system is $\mathrm{N}$-coupling. With $\mathrm{N}$ coupling, a user transaction that updates the quantity on hand for a part will cause HiPAC to schedule the condition-check for the rule to be performed as a separate transaction. Then, if this condition-checking transaction detects that the part is both critical and running low, the part-ordering action will be

\footnotetext{
${ }^{6}$ The language used to express the rule in our example is not the actual HiPAC rule language; it is simply an informal, relational calculus-like language intended to convey the flavor of ECA rules. Identifiers preceded with $\$$ are variables used to capture and pass values from one part of a rule to another.
}

scheduled as a third, separate transaction. These scheduling decisions are reliable in the sense that the system guarantees that the condition will be checked eventually (despite system failures) and that the action will eventually be performed if the condition is indeed found to be satisfied. However, all rule-related processing takes place outside the user transaction (and only if it completes successfully). $\mathrm{N}$-coupling's advantage is therefore that it minimizes both the impact of the rule on user transactions and the length of rule-related transactions; however, it can potentially permit the placement of multiple orders in this example (if several orders for the same critical, low-quantity part occur within a sufficiently short time period).

A rare possibility of multiple orders may be tolerable in some applications, but other applications may deem it intolerable. In the latter case, HiPAC's $C A$-coupling mode can be used to prevent double-ordering. With this coupling mode, the condition-check and the action (if needed) take place as a single, separate transaction; again, this transaction is scheduled (reliably) by a user transaction if it updates the quantity on hand of a part. As in the fully decoupled (i.e., N-coupled) case, CA-coupling adds no significant rule-related processing to the user transaction. And, since the condition-check and the action occur together as a single transaction in this case, it is guaranteed that double orders will not be placed. However, it is possible for potentially long delays to occur between the update to the quantity on hand and the subsequent placement of an order, as might happen if the system is heavily loaded.

If the needs of the application dictate that the placement of an order is a prerequisite for the successful delivery of a critical part which is running low, then the entire' execution of the rule must take place as part of the user transaction. In this case, HiPAC's strictest coupling mode, S-Coupling, must be specified for the rule. With this coupling mode, HiPAC will ensure that the delivery of the part (which occurs in the initial user transaction) will occur only if the ordering action, if required, is performed successfully.

\section{REFERENCES}

[1] R. Abbott and H. Garcia-Molina, "Scheduling real-time transactions: A performance evaluation," in Proc. 14th VLDB Conf., Los Angeles, CA, Aug. 1988.

[2] Aug. "Scheduling real-time transactions with disk-resident data," in Proc. 15th VLDB Conf., Amsterdam, The Netherlands, Aug. 1989.

[3] R. Agrawal and N. R. Gehani, "ODE (Object Data and Environment): The language and the data model," in Proc. ACM SIGMOD Int. Conf. Management Data, Portland, OR, 1989.

[4] J. A. Blakeley, P-A. Larson, and F. W. Tompa, "Efficiently updating materialized views," in Proc. ACM SIGMOD Int. Conf. Management Data, Washington, DC, 1986.

[5] A.P. Buchmann et al., "Time-critical database scheduling: A framework for integrating real-time scheduling and concurrency control," in Proc. 5th Int. Conf. Data Eng., Los Angeles, CA, 1989.

[6] P. Buneman and E. Clemens, "Efficiently monitoring relational databases," ACM Trans. Database Syst., Sept. 1979.

[7] M. Carey and M. Livny, "Distributed concurrency control performance: A study of algorithms, distribution, and replication," in Proc. 14th VLDB Conf., Los Angeles, CA, Aug. 1988.

[8] M. Carey, R. Jauhari, and M. Livny, "Priority in DBMS resource scheduling," in Proc. 15th VLDB Conf., Amsterdam, The Netherlands, Aug. 1989.

[9] U. Dayal, A. Buchmann, and D. McCarthy, "Rules are objects too: A knowledge model for an active, object-oriented database management system," in Proc. 2nd Int. Workshop Object-Oriented Database Syst., Bad Muenster am Stein, Ebernburg, West Germany, Sept. 1988. 
[10] U. Dayal et al., "HiPAC: A research project in active, time-constrained database management, (Final Technical Report)," Tech. Rep. XAIT89-02, XAIT Ref. 187, Xerox Advanced Information Technology, Cambridge, MA, July 1989

[11] D. Duchamp, "Analysis of transaction management performance," in Proc. 12th ACM Symp. Oper. Syst. Principles, Litchfield Park, AZ, Dec. 1989.

[12] K.P. Eswaran and D.D. Chamberlain, "Functional specifications of a subsystem for data base integrity," in Proc. 1st VLDB Conf., Framingham, MA, Sept. 1975.

[13] K.P. Eswaran, "Specifications, implementations, and interactions of a trigger subsystem in an integrated data base system," IBM Res. Rep. RJ1820, Aug. 1976.

[14] J. Gray, "Notes on database operating systems," in Operating Systems. An Advanced Course, R. Bayer, R. Graham, and G. Seegmuller, Eds. Berlin, Germany: Springer-Verlag, 1979.

[15] E. Hanson, "A performance analysis of view materialization strategies," in Proc. ACM SIGMOD Int. Conf. Management of Data, San Francisco, CA, 1987.

[16] M. Hsu, R. Ladin, and D. McCarthy, "An execution model for active data base management systems," in Proc. 3rd Int. Conf. Data Knowledge Bases, Jerusalem, Israel, June 1988.

[17] S. Hudson and R. King, "Cactis: A self-adaptive, concurrent implementation of an object-oriented database management system," ACM Trans. Database Syst., vol. 14, no. 3, Sept. 1989.

[18] R. Jauhari, M. Carey, and M. Livny, "Priority-hints: An algorithm for priority-based buffer management," in Proc. 16th VLDB Conf., Brisbane Australia, Aug. 1990.

[19] A. M. Kotz, K. R. Dittrich, and J. A. Mulle, "Supporting semantic rules by a generalized event/trigger mechanism," in Proc. Int. Conf. Extending Database Technol., Venice, Italy, Mar. 1988.

[20] B. Lindsay, L. Haas, and C. Mohan, "A snapshot differential refresh algorithm," in Proc. ACM SIGMOD Int. Conf. Management Data, Washington, DC, 1986

[21] B. Liskov, D. Curtis, P. Johnson, and R. Scheifler, "Implementation of Argus," in Proc. 11th ACM Symp. Oper. Sys. Principles, Austin, TX, Nov. 1987

[22] M. Livny, DeNet User's Guide, Version 1.0, Dep. Comput. Sci., Univ of Wisconsin, Madison, WI, 1988.

[23] D. McCarthy and U. Dayal, "The architecture of an active data base management system," in Proc. ACM SIGMOD Int. Conf. Management Data, Portland, OR, 1989.

[24] M. Morgenstern, "Active databases as a paradigm for enhanced computing environments," in Proc. 9th VLDB Conf., Florence, Italy, 1983.

[25] L. Raschid and S.Y.W. Su, "A transaction oriented mechanism to control processing in a knowledge base management system," in Proc. 2nd Int. Conf. Expert Database Syst., Tyson's Corner, VA, 1988.

[26] D. Rosenkrantz, R. Stearns, and P. Lewis, "System level concurrency control for distributed database systems," ACM Trans. Database Syst. vol. 3, no. 2, June 1978.

[27] N. Roussopoulos, "View indexing in relational databases," ACM Trans. Database Syst., vol. 7, no. 2, June 1982

[28] T.K. Sellis, C.-C. Lin, and L. Raschid, "Implementing large production systems in a DBMS environment: Concepts and algorithms," in Proc. ACM SIGMOD Int. Conf. Management Data, Chicago, IL, 1988

[29] J. Stankovic and W. Zhao, "On real-time transactions," Special issue on Real-Time Data Base Systems, SIGMOD Record, vol. 17, no. 1, Mar. 1988.

[30] M. Stonebraker, "Triggers and inference in database systems," in On Knowledge Base Management Systems, Brodie and Mylopoulos, Eds. Berlin, Germany: Springer-Verlag, 1986

[31] M. Stonebraker and L. Rowe, Eds., The POSTGRES Papers, Memorandum UCB/ERL M86/85, Electronics Research Laboratory, Univ. of California, Berkeley, CA, 5 Nov. 1986.

[32] M. Stonebraker, Ed., Readings in Database Systems. Los Altos, CA Morgan-Kaufman, 1988, pp. 503-506.

[33] M. Stonebraker et al., "The POSTGRES Rules System," IEEE Trans. Software Eng. Dec. 1988
[34] Y. Tay, J. Goodman, and R. Suri, "Locking performance in centralized databases," ACM Trans. Database Syst., vol. 10, no. 4, Dec. 1985.

[35] A. Tzvieli, "On the coupling of a production system shell and a DBMS," in Proc. 3rd Int. Conf. Data Knowledge Bases, Jerusalem, Israel, June 1988

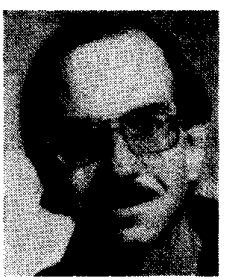

Michael J. Carey (S'77-M'83) received the B.S degree in electrical engineering and mathematics and the M.S. degree in electrical engineering (computer engineering) from Carnegie Mellon University in 1979 and 1981, respectively, and the Ph.D. degree in computer science from the University of California, Berkeley, in 1983.

Since then he has been on the Computer Sciences Department faculty at the University of Wisconsin-Madison, where he is currently an Associate Professor. He spent the summer of 1989 as a Visiting Scientist at IBM Almaden Research Center. His research interests include concurrency control performance, distributed databases, extensible and objectoriented database systems, and database support for real-time environments. $\mathrm{He}$ is a Co-Principal Investigator of the EXODUS extensible DBMS project.

Dr. Carey received an IBM Faculty Development Award in 1984, an Incentives for Excellence Award from Digital Equipment Corporation in 1986 , and an NSF Presidential Young Investigator Award in 1987. He is a member of the Association for Computing Machinery, an Associate Editor of IEEE DATA ENGINEERING, and Secretary/Treasurer of the ACM SIGMOD group.

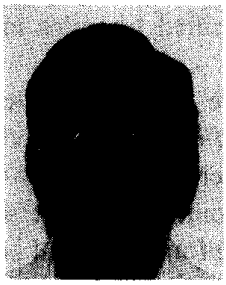

Rajiv Jauhari (S'88-M'90) received the B.Tech degree in electrical engineering in 1985 from the Indian Institute of Technology, Delhi.

$\mathrm{He}$ is currently working towards the Ph.D. degree in Computer Sciences at the University of Wisconsin-Madison. His research interests include modeling and performance evaluation of computer systems, priority scheduling algorithms for database systems, real-time database systems, and active databases.

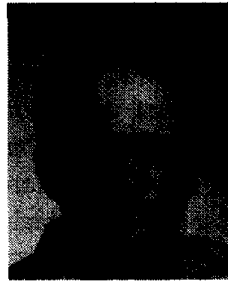

Miron Livny received the B.S. degree in physics and mathematics in 1975 from the Hebrew University and the M.Sc. and Ph.D. degrees in computer science from the Weizmann Institute of Science in 1978 and 1984, respectively.

Since 1983 he has been on the Computer Sciences Department faculty at the University of Wisconsin-Madison, where he is currently an Associate Professor. He has been a Visiting Scientist at IBM T.J. Watson Research Center and has consulted for a number of companies. His research focuses on performance evaluation of computer systems. His distributed database interests include concurrency control performance, data partitioning tradeoffs, load balancing, and real-time database systems. Other research interests include performance of distributed computing systems, distributed scheduling, resource management, and simulation tools. He was the developer of the DeNet simulation language, which is in use at a number of companies and universities. 